Pontifícia Universidade Catálica $_{\text {Do Rio DE JANeiro }}$

Marcos Cohen

\title{
Avaliação do Uso de Estratégias Colaborativas na Gestão de Unidades de Conservação do Tipo Parque na Cidade do Rio de Janeiro
}

Tese de Doutorado

Tese apresentada como requisito parcial para obtenção do título de Doutor pelo Programa de Pós-Graduação em Administração da PUC-Rio.

Orientador: Prof. Jorge Ferreira da Silva Co-orientadora: Profa. Alessandra Magrini

Rio de Janeiro

Abril de 2007 


\section{Pontifícia Universidade Católica \\ DO RIO DE JANEIRO}

Marcos Cohen

\section{Avaliação do Uso de Estratégias Colaborativas na Gestão de Unidades de Conservação do Tipo Parque na Cidade do Rio de Janeiro}

Tese apresentada como requisito parcial para obtenção do título de Doutor pelo Programa de Pós-Graduação em Administração da PUC-Rio. Aprovada pela Comissão Examinadora abaixo assinada.

Prof. Jorge Ferreira da Silva Orientador

Departamento de Administração - PUC-Rio

Prof. Paulo Cesar de Mendonça Motta Departamento de Administração - PUC-Rio

Profa. Angela Maria Cavalcanti da Rocha COPPEAD- UFRJ

Prof. Luiz Felipe Guanaes Rego Departamento de Geografia - PUC-Rio

Profa. Letícia Moreira Casotti UFRJ

Prof. João Pontes Nogueira Coordenador Setorial do Centro de Ciências Sociais - PUC-Rio 
Todos os direitos reservados. É proibida a reprodução total ou parcial do trabalho sem autorização da universidade, do autor e do orientador.

\section{Marcos Cohen}

Professor e Supervisor de Área do Departamento de Administração da PUC-Rio. Mestre em Administração de Empresas pela PUCRio, com especialização em Marketing. Engenheiro de Produção pela UFRJ. Atuou profissionalmente como: coordenador e gerente de estudos de mercado da Diretoria de Marketing Estratégico da Embratel; assessor da Diretoria Financeira e coordenador de projetos organizacionais da Cia. Metalúrgica Bárbara (atual Saint Gobain Canalizações); gerente de planejamento de materiais da Folic; consultor de empresas sênior da Andersen Consulting (atual Accenture).

Ficha Catalográfica

\begin{tabular}{|l} 
Cohen, Marcos \\
Avaliação do uso de estratégias colaborativas na \\
gestão de unidades de conservação do tipo parque na \\
cidade do Rio de Janeiro / Marcos Cohen ; orientador: \\
Jorge Ferreira da Silva ; co-orientadora: Alessandra Magrini. \\
-2007. \\
$\quad 341 \mathrm{f.} ; 30 \mathrm{~cm}$ \\
Tese (Doutorado em Administração)-Pontifícia \\
Universidade Católica do Rio de Janeiro, Rio de Janeiro, \\
2007. $\quad$ Inclui bibliografia \\
1. Administração - Teses. 2. Meio ambiente. 3. \\
Estratégias colaborativas. 4. Unidades de conservação. 5. \\
Parques. Gestão participativa. 7. Desenvolvimento \\
sustentável. 8. Parcerias. I. Silva, Jorge Ferreira da. II. \\
Magrini, Alessandra. Ill. Pontifícia Universidade Católica do \\
Rio de Janeiro. Departamento de Administração. Ill. Título. \\
\end{tabular}

CDD: 658 
A meus queridos Pais, pelo amor infindável e sacrifícios feitos para que eu pudesse chegar até aqui! 


\section{Agradecimentos}

Ao meu orientador, Prof. Jorge Ferreira da Silva, que durante estes doze anos de convívio na PUC-Rio, sempre me apoiou e confiou em meu trabalho.

À Profa. Alessandra Magrini, pela paciência e precisas contribuições à realização da pesquisa.

Aos professores do Departamento de Administração da PUC-Rio que me auxiliaram nessa difícil escalada.

Às caras amigas e colegas de turma Silvia Becher-Costa e Katia Rousseau, pelo companheirismo e ajuda nas horas difíceis.

Às caras amigas e colegas de Doutorado Lenise Saraiva de Vasconcelos Costa e Graziela Fortunato, sempre prontas a ajudar.

À secretária do Departamento Maria Teresa Campos, por quebrar todos os galhos do mundo, sempre com um sorriso nos lábios.

À Mônica Sztern, companheira nas boas e nas más horas.

À Maria de Lourdes Figueira, pela ajuda na pesquisa do PARNA da Tijuca.

À Secretaria Municipal de Meio Ambiente

Ao IEF-RJ

Ao IBAMA

Aos Gestores dos parques que participaram da pesquisa e que foram fundamentais para seu sucesso.

Aos atores sociais entrevistados durante a pesquisa, que contribuíram de forma significativa para a sua realização.

À Giorgia Werneck pela eficiência e gentileza na transcrição das entrevistas. 


\section{Resumo}

Cohen, Marcos; Silva, Jorge Ferreira da. Avaliação do uso de estratégias colaborativas na gestão de unidades de conservação do tipo parque na cidade do Rio de Janeiro. Rio de Janeiro, 2007. 341p. Tese de Doutorado - Departamento de Administração, Pontifícia Universidade Católica do Rio de Janeiro.

No ano de 2000 foi promulgada a lei 9985 que criou o Sistema Nacional de Unidades de Conservação Ambiental (SNUC) e regulamentou a gestão dos diferentes tipos de unidades de conservação ambiental no país, a fim de melhor protegê-las. Algumas das características inovadoras do SNUC são a definição de diretrizes e os mecanismos que assegurem a participação da sociedade, bem como a abertura para a colaboração de organizações nãogovernamentais, organizações privadas e pessoas físicas na criação, implantação e gestão dessas unidades. Na cidade do Rio de Janeiro, existem diversos tipos de unidades de conservação ambiental, sob responsabilidade de diferentes esferas do estado, porém as mais comuns são a do tipo parque. Trabalhos acadêmicos recentes, relatórios de auditorias realizados em alguns parques e a imprensa em geral têm mostrado que, apesar dos esforços empreendidos, os órgãos governamentais responsáveis vêm enfrentando dificuldades na implementação de uma gestão mais participativa, no estabelecimento de parcerias e na garantia da sustentabilidade desses parques. Nesse contexto, foi realizada uma pesquisa exploratória, de caráter qualitativo, com o objetivo de entender como as estratégias colaborativas utilizadas pelos responsáveis pelas unidades de conservação do tipo parque vêm sendo implementadas e como afetam os resultados da gestão e o relacionamento com diferentes grupos de atores sociais envolvidos, buscando, ao mesmo tempo, identificar os problemas vivenciados pelos gestores e os fatores intervenientes que facilitam ou dificultam sua atuação. Foram realizados múltiplos estudos de caso de unidades de conservação do tipo parque no Rio de Janeiro, sendo seis parques municipais, um parque estadual e um parque federal. Os dados foram coletados junto aos órgãos gestores dos parques e a representantes dos principais grupos de atores sociais envolvidos ou interessados na gestão desses parques. Realizaram-se pesquisas documentais, observações diretas e entrevistas semi-estruturadas. Após a análise dos casos individuais procedeu-se a uma análise cruzada dos casos para identificar padrões de estratégias, de resultados alcançados e de 
problemas comuns a todos eles. As conclusões indicam que os órgãos dos governos responsáveis vêm tomando iniciativas para estabelecer uma gestão mais participativa, por meio de Conselhos Consultivos, porém a maioria dos parques não havia ainda implementado tais Conselhos durante a realização da pesquisa. Observou-se também que o processo de formação e condução dos Conselhos é ainda lento e deficiente, causando problemas entre gestores e atores sociais. A grande quantidade de atores sociais e a presença de complexos fatores, tais como, falta de recursos financeiros, pressões de agentes econômicos e políticos, violência e crescimento urbano desordenado são fatores agravantes. Os casos revelaram diferentes formas de parcerias com outras organizações, desde informais até complexas, envolvendo múltiplos atores e mais de uma esfera de governo. Porém, fatores como burocracia, falta de autonomia dos gestores e a não utilização de instrumentos de gestão adequados a essa nova realidade são empecilhos à expansão dessas estratégias. Ao final, apresenta-se uma modelagem das interações observadas, seguida de proposições de caráter teórico sobre como as estratégias colaborativas são influenciadas pelos diferentes fatores identificados e como elas afetam os resultados da gestão dos parques, visando futuras pesquisas.

\section{Palavras-chave}

Meio ambiente; Estratégias colaborativas; Unidades de conservação; Parques; Gestão participativa; Desenvolvimento sustentável; Parcerias. 


\section{Abstract}

Cohen, Marcos; Silva, Jorge Ferreira da. Evaluation of collaborative strategies used in the managing environmental parks in Rio de Janeiro. Rio de Janeiro, 2007. 341 p. PhD Dissertation. IAG / Departamento de Administração, Pontifícia Universidade do Rio de Janeiro.

The National System of Conservation Units was established in Brazil, in July 2000, through Federal Law 9985. This legislative instrument defined different categories of environmental conservation units, in attempt to preserve them and regulate their sustainable use and management. Among the main innovations it comprises, the main aspect that should be pointed out is the fact that it ensures the participation of members of society in general as well as the collaboration of non-governmental organizations, enterprises and individual community members in implementing and managing such units. The city of Rio de Janeiro encompasses different types of conservation units, reporting to different governmental spheres; however, most of them belong to the category of parks. Recent academic studies, reports from auditing organizations that analyzed some of these parks and the press itself have revealed that state institutions have not been successful in their efforts of implementing more collaborative and participative management, in establishing productive partnerships and in assuring the sustainability of these parks. The present exploratory research of qualitative nature aims at analyzing the implementation of collaborative strategies in environmental parks and their effect on the results obtained by management, as well as the nature of the relationships set with the different social players involved. Management problems and the intervening factors that have helped or hindered the sustainability and conservation of such parks are also studied. Multiple case studies were used in the study: six municipal conservation parks managed by the city of Rio de Janeiro, one state park owned by the state of Rio de Janeiro and one federal park. Data were collected from the managing departments of those parks and from representatives from the different categories of stakeholders, including not only those involved directly in managing the parks but also those interested in the parks as users. Based on documentation, direct observation and semistructured interviews, the cases were analyzed individually and collectively, through cross-analysis, to identify common patterns of strategy implementation, successful practices and underlying problems. The findings revealed that 
governmental agencies have been making an effort to establish more participative management by means of councils, yet these management boards had not actually been implemented at the time of this research. The process of organizing and conducting such councils is slow and inefficient, and can cause controversies among social players and the management teams. The diversity of social actors and intervening factors such as lack of funds, economic and political pressures, uncontrollable urban growth and violence can be aggravating issues. The cases have shown different forms of partnership maintained with varied organizations - ranging from informal to complex; involving multiple actors and even different governmental powers. Factors such as bureaucracy, lack of management autonomy and the inadequate use of managing tools can be barriers to the expansion of such collaborative strategies. As a result of the findings, it was possible to devise a model of the interactions disclosed in the cases studied and a series of theoretical principles to explain how collaborative strategies are influenced by the different factors and how these strategies can affect the success of park management procedures. This model can be groundwork for future research.

\section{Key-Words}

Environment; Collaborative strategies; Conservation units; Parks; Participative management; Sustainable development; Partnerships 


\section{Sumário}

1 Introdução 20

1.1. O Desenvolvimento Sustentável Local e as Unidades de Conservação 20

1.2. Entendimento e Definição do Problema da Pesquisa 23

1.3. Principais Objetivos da Pesquisa 32

1.4. Delimitação da Pesquisa 33

1.5. Relevância da Pesquisa 33

2 Referencial Teórico $\quad 35$

2.1. Evolução do Pensamento e da Legislação Ambiental 38

2.2. Gestão Ambiental Pública e Legislação Ambiental 43

2.2.1. Gestão Ambiental Pública 43

2.2.2. Legislação Ambiental e Lei do Sistema Nacional de Unidades de Conservação 46

2.3. Conflitos Ambientais Urbanos 52

2.4. Estratégias Colaborativas e sua Aplicação ao Caso de Unidades de Conservação Ambiental $\quad 55$

2.4.1. Alianças e Redes de Colaboração $\quad 55$

2.4.2. Colaboração nas Questões Ambientais $\quad 59$

2.4.3. Enfoque Institucionalista 63

2.4.4. Críticas à Colaboração 65

2.4.5. Experiências com Gestão Participativa em Unidades de Conservação 66

2.5. Identificação dos Principais Atores Sociais Envolvidos com a Gestão de Unidades de Conservação Ambiental $\quad 70$

$\begin{array}{ll}\text { 2.5.1. O Estado } & 71\end{array}$

2.5.2. Comunidades Urbanas $\quad 74$

2.5.3. Organizações Não Governamentais (ONGs) 77

2.5.4. Empresas $\quad 79$

2.5.5. Órgãos de Financiamento 81

2.6. Discussão dos Resultados da Pesquisa Bibliográfica 82

3 Metodologia $\quad 84$ 
3.1. Estruturação Geral da Pesquisa 84

3.2. Premissas Filosóficas da Pesquisa 85

3.2.1. Alternativas de Paradigmas de Pesquisa 85

3.2.2. Decisão sobre o Paradigma de Pesquisa $\quad 87$

3.3. Definições Estratégicas da Pesquisa 89

3.4. Método de Pesquisa 94

3.4.1. Elaboração do Pré-Projeto da Pesquisa 94

3.4.2. Elaboração do Projeto de Pesquisa 95

3.4.3. Aprovação e Revisão do Projeto de Pesquisa 95

3.4.4. Desenho Conceitual e Elaboração do Protocolo de Estudo de Caso 96

3.4.4.1. Desenho Conceitual do Estudo de Caso 96

3.4.4.2. Protocolo dos Estudos de Caso 98

3.5. Limitações do Método 104

4 Apresentação e Análise Individual dos Estudos de Caso 106

4.1. Os Casos dos Parques Naturais Municipais 106

4.1.1. A Secretaria Municipal de Meio Ambiente 106

4.1.1.1. Breve Histórico do Sistema Municipal de Meio Ambiente 106

4.1.1.2. Atribuições e Organização da SMAC 107

4.1.1.3. Análise das Estratégias Centralizadas para Gestão dos Parques 111

4.1.2. Estudo de Caso do PNM Chico Mendes 129

4.1.2.1. Caracterização do Parque 129

4.1.2.2. Identificação dos Atores Sociais Locais 131

4.1.2.3. Análise da Gestão e das Estratégias Utilizadas para Atender ao SNUC 136

4.1.3. Estudo de Caso do PNM de Marapendi 146

4.1.3.1. Caracterização do Parque 146

4.1.3.2. Identificação dos Atores Sociais Locais 147

4.1.3.3. Análise da Gestão e das Estratégias Utilizadas para Atender ao SNUC 151

4.1.4. Estudo de Caso do PNM da Prainha 160

4.1.4.1. Caracterização do Parque 160

4.1.4.2. Identificação dos Atores Sociais Locais 161

4.1.4.3. Análise da Gestão e das Estratégias Utilizadas para Atender ao SNUC 163

4.1.5. Estudo de Caso do PNM Bosque da Barra 172 
4.1.5.1. Caracterização do Parque 172

4.1.5.2. Identificação dos Atores Sociais Locais 174

4.1.5.3. Análise da Gestão e das Estratégias Utilizadas para Atender ao SNUC 176

4.1.6. Estudo de Caso do PNM do Penhasco Dois Irmãos 187

4.1.6.1. Caracterização do Parque 187

4.1.6.2. Identificação dos Atores Sociais Locais 187

4.1.6.3. Análise da Gestão e das Estratégias Utilizadas para Atender ao SNUC 191

4.1.7. Estudo de Caso do PNM do Bosque da Freguesia 199

4.1.7.1. Caracterização do Parque 199

4.1.7.2. Identificação dos Atores Sociais Locais 200

4.1.7.3. Análise da Gestão e das Estratégias Utilizadas para Atender ao SNUC 203

4.1.8. Estudo de Caso do Parque Estadual da Pedra Branca 220

4.1.8.1. O Instituto Estadual de Florestas (IEF-RJ) 220

4.1.8.2. Caracterização do Parque 221

4.1.8.3. Identificação dos Atores Sociais Locais 223

4.1.8.4. Análise da Gestão e das Estratégias Utilizadas para Atender ao SNUC 225

4.1.9. Estudo de Caso do Parque Nacional da Tijuca 246

4.1.9.1. O IBAMA 246

4.1.9.2. Caracterização do Parque 246

4.1.9.3. Identificação dos Atores Sociais Locais 247

4.1.9.4. Análise da Gestão e das Estratégias Utilizadas para Atender $\begin{array}{ll}\text { ao SNUC } & 249\end{array}$

5 Análise entre Casos e Discussão dos Resultados 261

5.1. Gestão Participativa das Unidades de Conservação do Tipo Parque 262

5.1.1. Formação e Condução do Conselho Consultivo 262

5.1.2. Associações de Amigos de Parques e Outras Parcerias Informais 265

5.2. Colaboração com ONGs, Empresas e Instituições 268

5.2.1. Colaboração para Recuperar e Conservar os Ecossistemas 271

5.2.2. Colaboração em Educação Ambiental 272

5.2.3. Colaboração em Pesquisas Científicas 273

5.2.4. Colaboração para Organizar Atividades de Lazer e Ecoturismo 274 
5.3. Formas Alternativas de Gestão 275

5.3.1. Gestão Compartilhada 275

5.3.2. Gestão em Mosaico 276

5.4. Fatores de Sucesso para a Gestão dos Parques 277

6 Conclusões $\quad 279$

6.1. Conclusões à Luz dos Objetivos da Pesquisa 279

6.2. Modelo Conceitual do Problema 283

6.2.1. Proposições do Modelo 287

6.3. Considerações Finais 288

6.3.1. Reflexões sobre o Futuro 289

7 Glossário 290

8 Referências Bibliográficas 306

9 Anexos 308

9.1. Anexo 1 - Mapa das Unidades de Conservação do tipo Parque na cidade do Rio de Janeiro 308

9.2. Anexo 2 - Carta de Apresentação do Pesquisador 309

9.3. Anexo 3 - Listas de Questões para Entrevistas 310

9.4. Anexo 4 - Listagem Codificada das Entrevistas, Reuniões e $\begin{array}{ll}\text { Documentos da Pesquisa } & 319\end{array}$

9.5. Anexo 5 - Primeira Página do Termo de Cooperação entre SMAC e Carvalho Hosken para Adoção do PNM Prof. Mello Barreto 324

9.6. Anexo 6 - Mapa e Foto Aérea do PNM Chico Mendes 325

9.7. Anexo 7 - Mapa e Foto Aérea do PNM de Marapendi 326

9.8. Anexo 8 - Notícias sobre Poluição das lagoas da Barra da Tijuca Fonte: SERLA (2007) 327

9.9. Anexo 9 - Listagem da Fundação Parques e Jardins comprovando a adoção de uma área do PNM de Marapendi pela Associação do Condomínio Mundo Novo 331

9.10. Anexo 10 - Mapa e Foto Aérea do PNM Prainha 332

9.11. Anexo 11 - Folha de instauração do Inquérito Civil contra Autoridades e gestores do PNM da Prainha 333

9.12. Anexo 12 - Mapa e Foto Aérea do PNM Bosque da Barra 334

9.13. Anexo 13 - Mapa e Foto Aérea do PNM do Penhasco Dois Irmãos 335 
9.14. Anexo 14 - Mapa e Foto Aérea do PNM Bosque da Freguesia

9.15. Anexo 15 - Documentos relativos à Crise do Conselho Consultivo do PNM Bosque da Freguesia

9.16. Anexo 16 - Mapa e Foto Aérea do PE da Pedra Branca 


\section{Lista de figuras}

Figura 1- Modelo que representa o conceito de desenvolvimento sustentável

Figura 2 - Modelo lógico de inter-relação entre os conceitos estudados

Figura 4 - Os quatro paradigmas segundo Burrell e Morgan

(2003 (1979), p.3 )

Figura 5 - Fluxo das etapas e atividades da pesquisa

Figura 6 - Organograma da Secretaria Municipal de Meio Ambiente

(SMAC, 2005)

108

Figura 7 - Mapa da região mostrando os parques Chico Mendes

e Marapendi

Figura 8 - Área do PNM de Marapendi adotada pela Associação

Mundo Novo

Figura 10- Unidades de Conservação do Tipo Parque na cidade do

Rio de Janeiro

308

Figura 11 - Mapa do PNM Chico Mendes

325

Figura 12 - Foto com vista aérea do PNM Chico Mendes

325

Figura 13 - Mapa do PNM e da APA de Marapendi

326

Figura 14 - Foto com vista aérea do PNM Chico Mendes

326

Figura 15 - Mapa do PNM da Prainha

332

Figura 16 - Foto com vista aérea do PNM da Prainha

332

Figura 17 - Mapa do PNM Bosque da Barra

334

Figura 18 - Foto aérea do PNM Bosque da Barra

334

Figura 19 - Mapa do PNM do Penhasco Dois Irmãos

335

Figura 20 - Foto aérea do PNM do Penhasco Dois Irmãos

335

Figura 21 - Mapa do PNM Bosque da Freguesia

336

Figura 22 - Foto aérea do PNM Bosque da Freguesia

336

Figura 23 - Mapa do PE da Pedra Branca

340

Figura 24 - Núcleo da Lagoa do Camorim

340

Figura 25 - Mapa do PARNA da Tijuca mostrando três setores

do parque

Figura 26 - Vista aérea do setor da Pedra Bonita e Pedra da Gávea 


\section{Lista de tabelas}

Tabela 1- População Urbana e Rural no Brasil ao longo dos anos

(em milhares)

Tabela 2 -Estatísticas de denúncias de crimes ambientais

Tabela 3 - Estatísticas do IEF-RJ sobre o Progrma "Zero-Zero" ( IEF, 2007) 237 


\section{Lista de quadros}

Quadro 1 - Lista dos Parques existentes na cidade do Rio de Janeiro à época da pesquisa. 100

Quadro 2 - Motivos para seleção dos parques para os estudos de caso 101

Quadro 3 - Resumo das gravações e anotações de dados 102

Quadro 4 - Lista dos Parques Naturais Municipais selecionados para os estudos de caso 110

Quadro 5 - Mudanças de Governo e de gestão no IEF-RJ 225

Quadro 6 - Análise dos Conselhos Consultivos por Parque 263

Quadro 7 - Análise das Parcerias com Associações de Amigos do Parque e Parcerias informais $\quad 267$

Quadro 8 - Análise do alcance dos Objetivos de cada Parque por meio de colaboração 269

Quadro 9 - Resumo dos Fatores de Sucesso para gestão dos parques 278

Quadro 10 - Grau de alcance dos objetivos por esferas de governo 283 


\section{Lista das Siglas mais usadas}

AABF - Associação dos Amigos do Bosque da Freguesia

AMAF - Associação de Moradores e Amigos da Freguesia

AMCT - Associação dos Moradores do Canal das Taxas

AMOL - Associação dos Moradores da Orla da Lagoa da Barra da Tijuca

AMOR - Associação dos Moradores do Recreio dos Bandeirantes

APA - Área de Proteção Ambiental

ASAP - Associação dos Surfistas e Amigos da Prainha

CADI - Condomínios Associados Dois Irmãos

CAL - Associaçlão de Moradores do Alto Leblon

CEA - Centro de Educação Ambiental

CECA - Comissão Estadual de Controle Ambiental

CEDAE - Companhia Estadual de Águas e Esgotos

COMLURB - Companhia Municipal de Limpesa Urbana do Rio de Janeiro

CONSEMAC - Conselho Municipal de Meio Ambiente

FEEMA - Fundação Estadual de Engenharia do Meio Ambiente

FAFERJ - Federação das Associações de Moradores em Favelas do RJ

FPJ - Fundação Parques e Jardins do Rio de Janeiro

FUNBIO - Fundo Brasileiro para a Biodiversidade

GAE - Grupo de Ação Ecológico

GDA - Grupamento de Defesa Ambiental

GRUDE - Grupo de Defesa Ecológica

GUC - Gerência de Unidades de Conservação da SMAC

ha - Hectare

IBAMA - Instituto Brasileiro do Meio Ambiente e dos Recursos Naturais

Renováveis.

IBASE - Instituto Brasileiro de Análises Sociais e Econômicas

IEF-RJ - Instituto Estadual de Florestas do Rio de Janeiro

MMA - Ministério do Meio Ambiente

NIMA - Núcleo Intedisciplinar de Meio Ambiente da PUC-Rio

NIMA-JUR - Setor Jurídico do NIMA

ONG - Organização Não-Governamental

PARNA - Parque Nacional 
PCRJ - Prefeitura da Cidade do Rio de Janeiro

PNM - Parque Natural Municipal

REBEA - Rede Brasileira de Educação Ambiental

SEA - Secretaria de Estado de Ambiente do Rio de Janeiro (antiga SEMADS)

SEMADS - Secretaria Estadual de Meio Ambiente e Desenvovlimento

Sustentável do Rio de Janeiro

SEMA - Secretaria Federal do Meio Ambiente

SERLA - Fundação Superintendência Estadual de Rios e Lagoas

SMAC - Secretaria Municipal de Meio Ambiente

SNUC - Sistema Nacional de Unidades de Conservação

TCMRJ - Tribunal de Contas do Município do Rio de Janeiro 